\title{
Understanding the Ordering of Charged Nanoparticles in Water
}

\author{
Lopa Bhatt ${ }^{1}$, Nathan Rosenmann ${ }^{1}$, Jacob R. Jokisaari ${ }^{1}$, Mark Schlossman ${ }^{1}$ and Robert F. Klie ${ }^{1}$ \\ 1. University of Illinois at Chicago, Department of Physics, Chicago, IL, USA.
}

Charged nanoparticles have been useful in the treatments of diseases, such as cancer and bacterial infections as well as the removal of pollutants like oil or minerals from water [1]. Nevertheless, much remains unknown about the behavior of charged nanoparticles in aqueous solution. Since it is hard to image aqueous solution in a conventional transmission electron microscope, the study of nanoscale ordering of charged nanoparticle in water has been limited. This has been a barrier to the development of applications for nanoparticles.

New developments in the liquid cell sample holders has allowed us to image aqueous solution in scanning transmission electron microscope (STEM). In graphene or BN liquid cells, the solution that one wants to image is encapsulated between two mono-layers of materials, such as graphene or BN as shown in Figure $1[2,3]$. The focus of the work presented here is to examine charge screening of positive and negatively charged gold nanoparticles (+/- AuNP) in water with different concentrations of salt $(\mathrm{NaCl})$. Nanoparticle aggregation and ordering are studied using STEM and liquid cells fabricated with nanoparticles in various solutions.

Prior to making GLC's, dried solution of Au NPs was imaged in TEM to get an overall idea of how the particles are arranged. Then, if the concentration of the Au NP and salt in the solution was determined to be adequate, graphene liquid cells are used to examine the nanoparticles in water using the aberrationcorrected JEOL ARM200CF. Positively charged Au NPs have been examined in pure water and in solution with different concentrations of $\mathrm{NaCl}$.

The degree of aggregation is determined by measuring the radial distribution function (RDF) of a field of particles. The RDF is a measure of the interparticle potential and is used to compare images with high numbers of particles to examine how the distribution of particles differs with concentration of $\mathrm{NaCl}$. This information yields a much better understanding of the aggregation behavior of charged Au NP in water. The calculation of the RDF from the images using ImageJ will be discussed.

The preparation process of charged Au NPs in graphene liquid cells introduces some contamination that interferes with the analysis of nanoparticle solutions and can introduce additional charged species in the solution. Raman spectroscopy is a useful method to identify types of possible contaminates in the graphene liquid cells. Using Raman, we will demonstrate that cleaning steps using sulfuric acid and isopropyl alcohol help to reduce the concentration of contaminants in the graphene liquid cells. We will further show that the attraction between the nanoparticles and the graphene windows also poses a difficulty in reliably measuring the RDF. To reduce the attraction between the window material and nanoparticles, use of insulating window layers, such as $\mathrm{BN}$ will be discussed [4]. 


\section{References:}

[1] N de Jonge and FM Ross. Nature Nanotechnology 6 (2011), p. 695.

[2] JR.Jokisaari et al., Advanced Materials 30(36) (2018), p. 1802702.

[3] C Wang, T Shokuhfar and RF Klie, Advanced Materials 28(35) (2016), p. 7716.

[4] This work is supported in part by the UIC Chancellor's Undergraduate Research Award. This work made use of Electron Microscopy Services at Research Resources Center, UIC
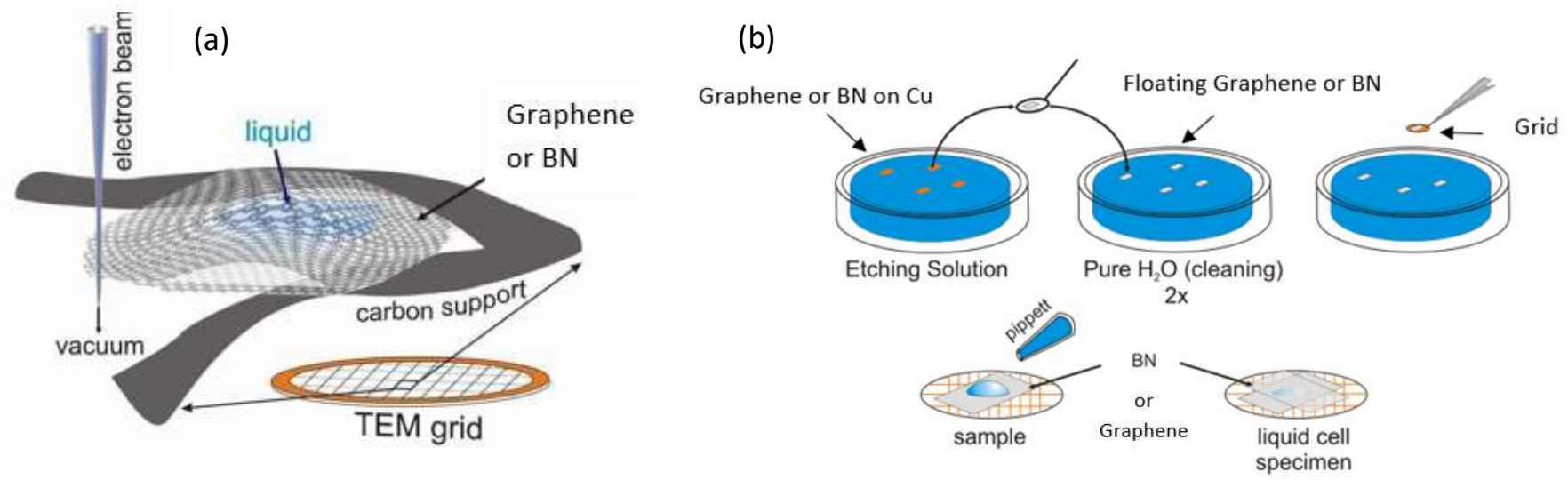

Figure 1. (a)Illustration of sample pockets in liquid cell

[2]. (b) Illustration of the process of making liquid cells

[2].

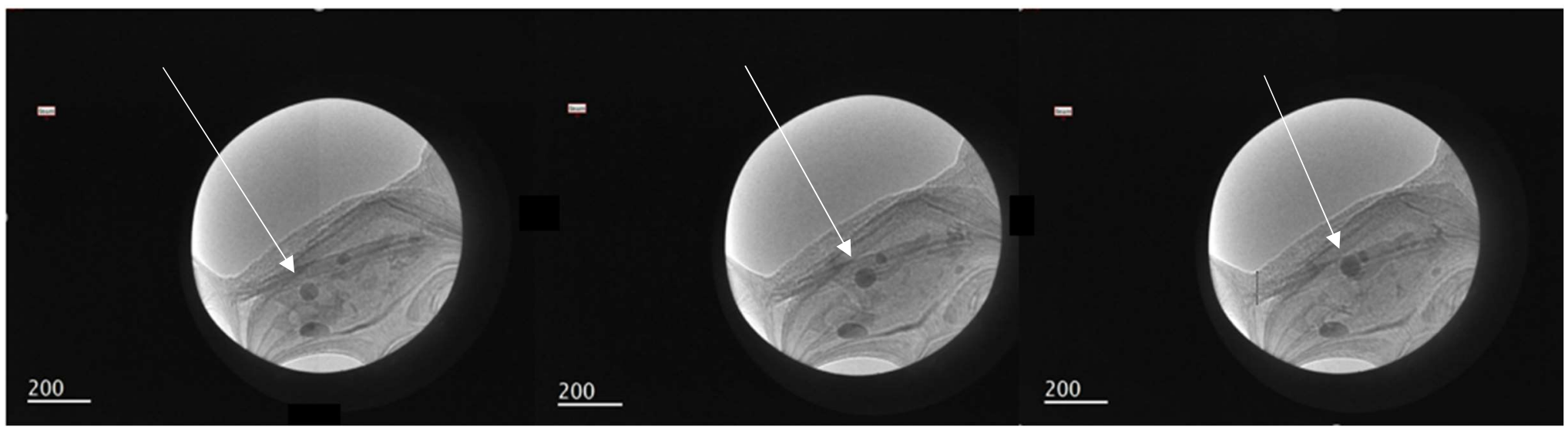

Figure 2. Motion of a negatively charged gold nanoparticle in a graphene liquid cell pocket 